\title{
The Schauder approach to degenerate elliptic equations with homogeneous Neumann boundary condition II
}

\author{
TOSHIO HORIUCHI*
}

\section{$\S 1$. Introduction}

Let $\Omega$ be a bounded domain in $\mathbf{R}^{n}(n \geq 2)$ with $\partial \Omega \in C^{\infty}$. Let $\varphi$ be a given nonnegative smooth function on $\bar{\Omega}$ and equivalent to a distance to the boundary:

$$
\Omega=\{\varphi(x)>0\}, \quad \partial \Omega=\{\varphi(x)=0\}, \quad d \varphi \neq 0 \text { on } \partial \Omega .
$$

For a nonnegative integer $k$ and a $\mu \in(0,1)$, by $C^{k+\mu}(\bar{\Omega})$ we denote the set of all functions on $\Omega$ whose $k$-th order partial derivatives are uniformly Hölder continuous with exponent $\mu$. Let $\alpha(x)$ be an arbitrary real-valued function of class $C^{1+\mu}(\bar{\Omega})$ and let $c(x)$ be of class $C^{\mu}(\bar{\Omega})$. Then we set

$$
\mathcal{P} u=-\operatorname{div}\left(\varphi(x)^{\alpha(x)} \nabla u\right)+\varphi(x)^{\alpha(x)} c(x) u .
$$

Here $\nabla u=\left(\frac{\partial u}{\partial x_{1}}, \frac{\partial u}{\partial x_{2}}, \cdots, \frac{\partial u}{\partial x_{n}}\right)$, and in particular if $\alpha(x) \equiv 0$, then $\mathcal{P} u \equiv$ $-\Delta u+c(x) u$.

The main purpose of this paper is to study the homogeneous Neumann boundary value problem defined by

$$
\begin{cases}\mathcal{P} u=f & \text { in } \Omega \\ \frac{d u}{d \nu}=0 & \text { on } \partial \Omega\end{cases}
$$

where by $\nu$ we denote the unit inward normal to $\partial \Omega$.

Under some assumptions on the operators $\mathcal{P}$, the existence and uniqueness of the classical solution of (N-P) will be shown for any given $f$ belonging to a certain Schauder space. Obviously (N-P) is a generalization of the classical Neumann problem for the uniformly elliptic operators to the wider class of operators $\mathcal{P}$ with a non-constant parameter $\alpha(x)$. Namely, the operators $\mathcal{P}$ may degenerate finitely in all directions on the set of the boundary points where $\alpha(x)>0$, and the degeneracy of $\mathcal{P}$ varies in accordance with the values of $\alpha(x)$. Furthermore, if $\alpha(x)<0$ on some subset of the boundary, then the coefficients of $\mathcal{P}$ do not remain bounded. As a result, it will be shown in $\S 2$ that $(\mathrm{N}-\mathrm{P})$ admits unbounded

Received February 6, 1996.

1991 Mathematics Subject Classification. Primary 35J70 Secondary 35J60, 35J20.

This research was partially supported by Grant-in-Aid for Scientific Research (08640163), Ministry of Education, Science, Sports and Culture.

* Department of Mathematical Sciences, Ibaraki University, Mito, Ibaraki 310, Japan. 
classical solutions, even if $f$ is regular and $\alpha$ is nonnegative. So, in order to do with these unbounded solutions we are forced to modify the classical Schauder spaces in a proper way ( See $\S 2, \S 6$ and Appendix A).

There are already authors who have studied the degenarate elliptic equations in the Hölder framework. Goulaouic-Shimakura [2] studied the equations degenerated in all derections at the boundary. In [5] the author studied the equations degenerated only in normal direction at each point of the boundary. In these works, no boundary condition was imposed because the so called " entrance property " of the boundary with respect to the operator was assumed. And Graham studied in [3] and [4] the Dirichlet problems for Bergman Laplacian in some Hölder spaces. Our interest is not only to study the operators $\mathcal{P}$ under the homogeneous Neumann boundary condition in some modified Schauder spaces, but also to indicate that the classical theory for uniformly elliptic differential operators can be extended to the degenerate operators $\mathcal{P}$ in a natural way. Our main method is, as in [2], [3], [4] and [5], to make use of the fundamental solution for some simplest model of our operators (See also [6] and [7]).

Although our aim is to study the Neumann problem (N-P), here we breafly remark about the homogeneous Dirichlet boundary problen with respect to a similar operator, which will be our starting point in this paper. Let us consider

$$
\begin{cases}-\operatorname{div}\left(\varphi^{\alpha(x)} \nabla u\right)+c(x) \varphi^{\alpha(x)-1} u=f & \text { in } \Omega \\ u=0 & \text { on } \partial \Omega .\end{cases}
$$

In (D-P), we impose auxiliarily on $\alpha$ the following condition (D).

$$
\frac{d \alpha}{d \nu}=0 \quad \text { on } \partial \Omega
$$

Then we have

Proposition.1-1. Suppose that $\alpha(x)$ is of cass $C^{2+\mu}(\bar{\Omega})$ and satisfies the condition (D). Moreover we suppose that $\alpha<1$ on $\partial \Omega$. Then there is a positive number $M$ such that if inf $c(x)_{\Omega} \geq M$ then for every $f \in C^{\mu}(\bar{\Omega})$, there exists one and only one solution $u$ to $(D-P)$ which is written as $u=\varphi^{1-\alpha} v$ with a function $v$ belonging to $C^{1+\mu}(\bar{\Omega})$ such that $\varphi v \in C^{2+\mu}(\bar{\Omega})$.

Proof OF PROPOSITION 1-1. From the theory of ordinary differential equations, we see that (D-P) has no solutions vanishing at $x_{n}=0$ if $\alpha \geq 1$. So the condition $\alpha<1$ is necessary for us. Now we set $u=\varphi^{1-\alpha} v$ in (D-P). Then, by virtue of the condition (D), this proposition follows as a corollary to the theorem due to C.Goulaouic-N.Shimakura [2] in which the equation $-\varphi \Delta v-z \partial_{n} v+F=0(F \in$ $C^{\mu}(\bar{\Omega}), \Re z>0$ ) were studied. We can apply their result to (D-P) putting $z=2-$ $\alpha$ and $F=-f+$ [ error terms arising from the change of unknown functions ].

Here we give an important remark: If $\alpha<0$, then the solution $u$ to the problem (D-P) obviously satisfies the homogeneous Neumann condition as well. But this $u$ is excluded from our framework for the problem (N-P) ( See also Example in the next section ). 
This paper is organized in the following way: Our main results concerning the Neumann problem (N-P) will be stated in $\S 2$. We also give the sketch of the proofs and some examples which indicate "the sharpness" of the results. In $\S 3$ we shall establish Theorem 2-1 admitting an a priori estimate of solutions in the half space in $\S 5$ and $\S 6$. Using Theorem 2-1. we give a proof to Theorem 2-2 in $\$ 4$. In $\S 5$ we define the Green function for the Neumann problem (N-P') and describe some fundamental properties. The sections 6 is devoted to establish the a priori estimate of solutions in the half space $\mathbf{R}_{+}^{n}$, which plays an essential role in the present paper. Various properties of the modified Schauder spaces, which are needed in the proofs of the theorems, will be proved in the Appendix.

\section{§2 Main Results}

In the Neumann problem, we have to deal with unbounded solutions as we shall show in the end of this section. So we are obliged to modify the classical Schauder spaces to admit unbounded solutions. To this end we define the function spaces represented by $\Lambda_{\tau}^{\mu}(\Omega)$ ( See also Definition 6-1 in $\S 6$ ).

Definition 2-1. Let $0<\mu<1, \tau \neq 0$. A function $u$ is said to belong to the class $\Lambda_{\tau}^{\mu}(\Omega)$ if

$$
\|u\|_{\mu, \tau}=|u|_{\mu, \tau}+\sup _{\Omega}\left|\varphi^{(-\tau)}+u\right|
$$

is finite, where

$$
|u|_{\mu, \tau}=\sup _{\Omega \times \Omega} \min \left(\varphi(x)^{(\mu-\tau)_{+}}, \varphi(y)^{(\mu-\tau)_{+}}\right) \frac{|u(x)-u(y)|}{|x-y|^{\mu}} .
$$

Here, $\tau_{+}=\max (\tau, 0)$ and $\tau_{-}=\min (\tau, 0)$. Also, $u$ is said to belong to the class $\Lambda_{2+\tau}^{2+\mu}(\Omega)$ if $D^{\gamma} u \in \Lambda_{\tau}^{\mu}(\Omega)$ for any $\gamma$ with $|\gamma| \leq 2$ and we denote $\|u\|_{2+\mu, 2+\tau}=$ $\sum_{|\gamma| \leq 2}\left\|\partial^{\gamma} u\right\|_{\mu, \tau}$.

Here we note that if $\tau \geq \mu$, then the class $\Lambda_{\tau}^{\mu}(\Omega)$ is reduced to the class $C^{\mu}(\bar{\Omega})$. As for these modified Schauder spaces, it holds that the interporlation inequalities of the following type (For the proof, see $\S$ Appendix ) :

Proposition A.

(1) Assume that $u \in \Lambda_{2+\tau}^{2+\mu}(\Omega), \tau>-1+\mu$. Then

$$
\sum_{|\gamma| \leq 1}\left\|\partial^{\gamma} u\right\|_{\mu, \mu} \leq C(\Omega)\left[\sum_{|\gamma|=2}\left\|\partial^{\gamma} u\right\|_{\mu, \tau}+\|u\|_{\mu, \tau}\right] .
$$

(2) Let $p \in \partial \Omega$ and $w_{R}=\Omega \cap\{x:|x-p|<R\}$ for a small $R>0$. Assume that $u \in \Lambda_{2+\tau}^{2+\mu}(\Omega), 0>\tau>-1+\mu$ and $\operatorname{supp} u \subset \omega_{R} \cup\left(\partial \omega_{R} \cap \partial \Omega\right)$. Then, for any $\varepsilon \in(0,-\tau)$, there is a positive number $C_{\varepsilon}$ such that we have

$$
\left\|\partial_{x}, u \log \varphi\right\|_{\mu, \tau} \leq C_{\varepsilon} R^{1-\varepsilon}\|u\|_{2+\mu, 2+\tau}, \quad \text { for } j=1,2, \ldots, n .
$$


(3) Assume that $u \in \Lambda_{\tau}^{\mu}(\Omega), \tau>-1+\mu, \tau \neq 0$. Then, for any $\varepsilon>0$, there is a positive number $C_{\varepsilon}$ such that

$$
\sum_{j=1}^{n}\left\|\partial_{x_{j}} u\right\|_{\mu, \tau} \leq \varepsilon \sum_{j, k=1}^{n}\left\|\partial_{x_{j} x_{k}}^{2} u\right\|_{\mu, \tau}+C(\varepsilon)\|u\|_{\mu, \tau} .
$$

Our goal is the theorem of existence and uniqueness of the solution to (N-P) in the function space $\Lambda_{2+\tau}^{2+\mu}$ (Theorem 2-2 below ). And this is relied upon an a priori inequality for solutions ( Theorem 2-1 below ) and the method of continuity. To obtain an a priori inequality, we proceed as follows. By a partition of unity of the closure $\bar{\Omega}$, the question is essentially reduced to prove an inequality for $u$ with small support in a neighborhood of a boundary point. This is because the operator is not degenerate in the interior of the domain so that a nice inequality in the interior is guaranteed by a classical result of J.Schauder. Let $x^{0}$ be any boundary point. Then, there exists a neighborhood $U$ of $x^{0}$ and diffeomorphism $\chi$ from $U \cap \bar{\Omega}$ in the $x$-space to a semi-ball $\left\{|y|<r, y_{n} \geq 0\right\}$ in the $y$-space such that $\chi\left(x^{0}\right)=0, \chi(U \cap \partial \Omega) \subset\left\{y_{n}=0\right\}$ and that $\varphi\left(\chi^{-1}(y)\right)=y_{n}+o\left(y_{n}\right)$. Clearly, $\alpha(x)$ is close to the constant function $\alpha\left(x^{0}\right)$ in $U$. Rewriting the new coordinates $y$ again by $x$, we can locally approximate (N-P) by a problem

$$
\begin{aligned}
& L_{\alpha} u \equiv-x_{n}^{\alpha} \Delta u-\alpha x_{n}^{\alpha-1} \partial_{x_{n}} u=f \quad \text { in } \mathbf{R}_{+}^{n}, \\
& \frac{\partial u}{\partial x_{n}}=0 \quad \text { on }\left\{x: x_{n}=0\right\}
\end{aligned}
$$

for $u$ with small support in a neighborhood of the origin. Here $\alpha$ is a constant satisfying $\alpha>-1$ and $f$ on the right hand side contains an error term arising from the localization.

Theorem 2-1. Assume that $\alpha(x)>-1$ on $\partial \Omega$. Let $\tau, \mu$ and $\alpha$ satisfy

$$
\max (-1,-1+\mu-\alpha(x))<\tau<0 \quad \text { on } \quad \partial \Omega .
$$

Let $u \in \Lambda_{2+\tau}^{2+\mu}(\Omega)$ be a solution of $(N-P)$ for $f / \varphi(x)^{\alpha(x)} \in \Lambda_{\tau}^{\mu}(\Omega)$. Then it holds that

$$
\|u\|_{2+\mu, 2+\tau}+\left\|\varphi^{-1}(\nabla \varphi, \nabla u)\right\|_{\mu, \tau} \leq C\left\|f / \varphi^{\alpha}\right\|_{\mu, \tau}+C^{\prime}\|u\|_{\mu, \tau}
$$

Here $C$ and $C^{\prime}$ are positive numbers independent of $f$ and $u$.

We get first the inequality for solutions to (N-P') with small support with the aid of Theorem 6-1 and estimate the error terms arising from the partition of unity. We need Proposition $A$ for an interpolation argument and to treat the terms involving $\log \varphi$. Finally, to prove Theorem 2-1, we sum up the both sides of a finite number of inequalities in the local coordinate neighborhoods.

The existence and uniqueness of the solution to (N-P), which is the main theorem in this article, is stated as follows : 
THEOREM 2-2. Assume that $\alpha(x)>-1$ on $\partial \Omega, c(x)>0$ on $\bar{\Omega}$ and that

$$
\max \left(-1+\mu,-1+\mu-\alpha(x),-\frac{1}{2},-\frac{\alpha(x)+1}{2}\right)<\tau<0 \quad \text { on } \quad \partial \Omega .
$$

Then, (N-P) has a unique solution in the space $\Lambda_{2+\tau}^{2+\mu}(\Omega)$ for every $f$ such that $f / \varphi^{\alpha} \in \Lambda_{\tau}^{\mu}(\Omega)$.

Theorem 2-2 is proved in the following way : First, we prove the uniqueness of the solution with the aid of a function space

$$
W(\Omega)=\left\{u: \int_{\Omega}\left(|\nabla u|^{2}+c|u|^{2}\right) \varphi^{\alpha} d x<\infty\right\} .
$$

Note that $f$ belongs to the dual space of $W(\Omega)$ provided that $\tau>-(\alpha(x)+1) / 2$ and $\alpha(x)>-1$ on $\partial \Omega$. Since the weak solution is unique in the space $W(\Omega)$, the classical solution belonging to $\Lambda_{2+\tau}^{2+\mu}(\Omega)$ is also unique if there exists any. Second, if $\alpha(x)$ is identically equal to 0 , then (N-P) is a variant of the classical Neumann problem for the Laplacian in a bounded domain. Lastly, for general $\alpha(x)$, we make use of the method of continuity. In each step of the reasoning, we need some restrictions on the values of $c(x), \alpha(x), \mu$ and $\tau$ (see (2-3) and (2-5) ).

REMARK. Theorems 2-1 and 2-2 are also valid for the following (N-P") if $\tau \neq 0$ (in place of $\tau<0$ ) and if $\inf _{\Omega} c(x)$ is sufficiently large.

$$
\left\{\begin{array}{l}
-\varphi^{\alpha} \Delta u-\alpha \varphi^{\alpha-1}(\nabla \varphi, \nabla u)+c(x) \varphi^{\alpha} u=f \quad \text { in } \Omega \\
\frac{d u}{d n}=0 \quad \text { on } \partial \Omega .
\end{array}\right.
$$

In the rest of this section, we give an example of unbounded solution to ( $\mathrm{N}$ P). For simplicity we consider (N-P) locally in some neighborhood of the origin of $\mathbf{R}_{+}^{n}$ in place of a general domain $\Omega$.

EXAMPLE. Let $\mathcal{Q}$ be the operator defined to be

$$
\mathcal{Q} u=-\operatorname{div}\left(x_{n}^{x_{1}} \nabla u\right)=-x_{n}^{x_{1}}\left[\Delta u+\frac{x_{1}}{x_{n}} \partial_{x_{n}} u+\left(\log x_{n}\right) \partial_{x_{1}} u\right] .
$$

Then, for $u=x_{n}^{2} \log x_{n}-2 x_{1}-x_{1}^{2}$, we have $\left.\frac{\partial u}{\partial x_{n}}\right|_{x_{n}=0}=0$ and $\mathcal{Q} u=x_{n}^{x_{1}}\left(x_{1}-\right.$ 1) $\in C^{\mu}$ if $x_{1}>\mu$. However $\partial_{x_{n}}^{2} u=3+2 \log x_{n} \notin C^{\mu}$. And we see that $u \in \Lambda_{2+\tau}^{2+\mu}\left(\mathbf{R}_{+}^{n} ; l o c\right)$ for any $\mu \in(0,1)$ and any $\tau<0$.

Note also that $w=x_{n}^{1-x_{1}}$ satisfies $L w=0$ with the homogeneous Dirichret condition $\left.w\right|_{x_{n}=0}=0$ if $x_{1}<1$ and the homogeneous Neumann condition $\left.\frac{\partial w}{\partial x_{n}}\right|_{x_{n}=0}=0$ if $x_{1}<0$ respectively. According to Proposition 1-1, we can consider $w$ as a null-solution to the Dirichret problem (See (D-P) with $c=0$ ). Moreover, we see that $w$ is excluded from our framework for the problem (N-P). To see this, let $K$ be a compact set contained in $\overline{\mathbf{R}_{+}^{n}} \cap\left\{x:-1<x_{1}<0\right\}$ such that $K \cap\left\{x: x_{n}=0\right\} \neq \phi$ and assume that $w \in \Lambda_{2+\tau}^{2+\mu}(K ; l o c)$ for some $\mu \in(0,1)$ and $\tau<0$. Then, we have $\frac{\partial^{2} w}{\partial x_{n}^{2}}=x_{1}\left(x_{1}-1\right) x_{n}^{-1-x_{1}} \in \Lambda_{\tau}^{\mu}(K ; l o c)$. Furthermore, it follows from the assertions (1) and (3) in Lemma A-2 that $\tau \leq \min _{x \in K \cap\left\{x_{n}=0\right\}}\left(-1-x_{1}\right)$. But this contradicts to the conditions (2-3) and $(2-5)$ in the main theorems, so that the assertion follows. 


\section{$\S 3$. The proof of Theorem 2-1.}

In this subsection we shall establish Theorem 2-1 using the results in the subsequent sections and Appendix. Though the function spaces and the degeneracy of the operators are rather different from those in [2] and [3], their arguements work in a similar way. Since the interpolation inequalities (Proposition A ) are valid, it suffices to prove the following weak inequality in place of (2-4).

LeMma 3-1. Assume that $\alpha(x)>-1$ on $\partial \Omega$. Let $\tau, \mu$ and $\alpha$ satisfy

$$
\max (-1,-1+\mu-\alpha(x))<\tau<0 \quad \text { on } \quad \partial \Omega .
$$

Let $u \in \Lambda_{2+\tau}^{2+\mu}(\Omega)$ be a solution of $(N-P)$ for $f / \varphi(x)^{\alpha(x)} \in \Lambda_{\tau}^{\mu}(\Omega)$. Then it holds that

$$
\begin{aligned}
\|u\|_{2+\mu, 2+\tau} & +\left\|\varphi^{-1}(\nabla \varphi, \nabla u)\right\|_{\mu, \tau} \\
& \leq C\left(\left\|f / \varphi^{\alpha}\right\|_{\mu, \tau}+\sum_{k=1}^{n}\left\|\partial_{x_{k}} u\right\|_{\mu, \tau}+\|u\|_{\mu, \tau}\right) .
\end{aligned}
$$

Here $C$ is a positive number independent of $f$ and $u$.

We begin with a localization. Let $\left\{\Omega_{k}\right\}_{k=0}^{N}$ be a family of an open set with a smooth boundary such that

$$
\overline{\Omega_{0}} \subset \Omega, \quad \bar{\Omega} \subset \cup_{k=0}^{N} \Omega_{k} .
$$

Let $\left\{\theta_{k}\right\}_{k=1}^{N}$ be a partition of unity subordinate to this open covering, namely,

$$
\theta_{k} \in C_{0}^{\infty}\left(\Omega_{k}\right) \quad \text { and } \quad \sum_{k=0}^{N} \theta_{k}=1 \quad \text { on } \bar{\Omega} .
$$

By a techinical reason we also impose on each $\theta_{k}$ the following condition:

$$
\left.\left(\nabla \varphi, \nabla \theta_{k}\right)\right|_{\partial \Omega}=0, \quad \text { for } k=1,2,3, \ldots, N .
$$

By virtue of a localization argument and the condition (3-3), it suffices to establish the inequality for each function $\theta_{k} u$ with $u \in \Lambda_{\tau}^{\mu}(\Omega)$ for $k=1,2, \ldots, N$. Let $U$ be one of the openset $\Omega_{k}(k=1,2, \ldots, N)$ and let $x_{0} \in U \cap \partial \Omega$. As has been already mensioned, by $\chi(x)=\left(\chi_{1}(x), \chi_{2}(x), \ldots, \chi_{n}(x)\right)$ we denote a smooth diffeomorphism such that

$$
\begin{aligned}
& \chi: U \cap \bar{\Omega} \longrightarrow \underline{W_{R}}=\left\{y:\left|y^{\prime}\right|<R, 0 \leq y_{n}<R\right\}, \\
& \chi(U \cap \partial \Omega) \subset\left\{y: y_{n}=0\right\}, \\
& \varphi\left(\chi^{-1}(y)\right)=y_{n}+o\left(y_{n}\right) \text { and } \chi\left(x_{0}\right)=0,
\end{aligned}
$$

and $\chi$ satisfies the equations on the boundary : 


$$
\left(\nabla \varphi, \nabla \chi_{j}\right)=\delta_{n, j}, \quad \text { on } \partial \Omega \cap U \quad \text { for } j=1,2, \ldots, n \text {. }
$$

By $f^{*}(y)$, we denote $f\left(\chi^{-1}(y)\right)$ for any function $f$. By virtue of this $\chi$, the operator $\mathcal{A}$ defined by

$$
\begin{aligned}
\mathcal{A} & =\frac{1}{\varphi(x)^{\alpha(x)}} \operatorname{div}\left(\varphi(x)^{\alpha(x)} \nabla \cdot\right)+c(x) \\
& =-\Delta-\frac{\alpha(x)}{\varphi(x)}(\nabla \varphi(x), \nabla \cdot)+(\nabla \alpha(x), \nabla \cdot) \log \varphi(x)+c(x),
\end{aligned}
$$

is transformed to

$$
\mathcal{B}=-\sum_{j, k=1}^{n} b_{j, k}^{*}(y) \partial_{y_{j}, y_{k}}^{2}-\sum_{k=1}^{n} b_{k}^{*}(y) \partial_{y_{k}}+b_{0}^{*}(y),
$$

where

$$
\begin{gathered}
b_{j, k}(x)=\left(\nabla \chi_{j}(x), \nabla \chi_{k}(x)\right), \quad b_{0}(x)=c(x), \\
b_{k}(x)=\frac{\alpha(x)}{\varphi(x)}\left(\nabla \varphi(x), \nabla \chi_{k}(x)\right)+\Delta \chi_{k}(x) \\
+\left(\nabla \alpha(x), \nabla \chi_{k}(x)\right) \log \varphi(x) .
\end{gathered}
$$

Here we note that

$$
\begin{aligned}
& b_{j, k}(x) \xi_{j} \xi_{k} \geq b_{0}|\xi|^{2}, \quad \text { for some number } b_{0}>0 \\
& \varphi^{*}(y)=y_{n}+o\left(y_{n}\right), \quad\left(\nabla \varphi, \nabla \chi_{k}\right)^{*}(y)=\delta_{k, n}+O\left(y_{n}\right) \\
& b_{k}^{*}(y)=\delta_{k, n} \frac{\alpha^{*}(y)}{y_{n}}+\left(\nabla \alpha, \chi_{k}\right)^{*}(y) \log y_{n}+h_{k}(y)
\end{aligned}
$$

where $h_{k}(y)$ is a bounded differentiable function.

Then Lemma 3-1 follows directly from the following local version on $\mathbf{R}_{+}^{n}$ :

Lemma 3-2. Assume that $\alpha(x)>-1$ on $\partial \Omega$. Let $\tau, \mu$ and $\alpha$ satisfy the inequality (2-3) in Theorem 2-1. Moreover we suppose that the operator $\mathcal{B}$ satisfies (3-8) and (3-9). Then, for sufficiently small $r \in(0, R / 2)$, there exists a positive number $C$ such that

$$
\begin{gathered}
\|u\|_{2+\mu, 2+\tau ; W_{r}}+\left\|\partial_{y_{n}} u / y_{n}\right\|_{\mu, \tau ; W_{r}} \\
\leq C\left[\|\mathcal{B} u\|_{\mu, \tau ; W_{2 r}}+\sum_{k=1}^{n}\left\|\partial_{y_{k}} u\right\|_{\mu, \tau ; W_{2 r}}+\|u\|_{\mu, \tau, W_{2 r}}\right], \\
\text { for any } u \in \Lambda_{2+\tau}^{2+\mu}\left(W_{R}\right) \cap \mathcal{E}^{\prime}\left(\underline{W_{R}}\right) .
\end{gathered}
$$


Here by $\|\cdot\|_{\mu, \tau ; S}$ and $\|\cdot\|_{2+\mu, 2+\tau ; S}$, we denote the norms defined by (6-3) on the spaces $\Lambda_{\tau}^{\mu}(S)$ and $\Lambda_{2+\tau}^{2+\mu}(S)$ respectively.

In order to establish this, we auxiliarily set

$$
\mathcal{B}_{0}=-\sum_{j, k=1}^{n} b_{j, k}^{*}(0) \partial_{y_{j}, y_{k}}^{2}-\frac{\alpha^{*}(0)}{y_{n}} \partial_{y_{n}}
$$

So that $\mathcal{B}=\mathcal{B}_{0}+\mathcal{B}_{1}+\mathcal{B}_{2}$, where

$$
\begin{aligned}
& \mathcal{B}_{1}=-\sum_{j, k=1}^{n}\left[b_{j, k}^{*}(y)-b_{j, k}^{*}(0)\right] \partial_{y_{j}, y_{k}}^{2}-\frac{\alpha^{*}(y)-\alpha^{*}(0)}{y_{n}} \partial_{y_{n}} \\
& \mathcal{B}_{2}=-\sum_{k=1}^{n}\left[\left(\nabla \alpha, \nabla \chi_{k}\right)^{*}(y) \log y_{n}+h_{k}(y)\right] \partial_{y_{k}}+b^{*}(y) .
\end{aligned}
$$

Since it follows from (3-5) that

$$
\left.b_{j, n}(x)\right|_{x \in \partial \Omega}=0, \quad j=1,2, \ldots, n-1,
$$

by a linear transformation in $\mathbf{R}^{n-1}$, the operator $\mathcal{B}_{0}$ is transformed to

$$
\mathcal{L}_{\alpha^{*}(0)} \equiv-\Delta-\frac{\alpha^{*}(0)}{y_{n}} \partial_{y_{n}}
$$

By $\omega_{R}$ we denote the image of $W_{R}$ by this transform. Then the following a priori estimate holds. satisfy

Proposition 3-1. Suppose that $R>0$ and $\alpha^{*}(0)>-1$. Let $\tau, \mu$ and $\alpha^{*}(0)$

$$
\max \left(-1,-1+\mu-\alpha^{*}(0)\right)<\tau
$$

Then, there is a positive constant $C$ such that for every $u \in \Lambda_{2+\tau}^{2+\mu}\left(\omega_{R}\right) \cap \mathcal{E}^{\prime}\left(\underline{\omega}_{R}\right)$, we have

$$
\begin{aligned}
\|u\|_{2+\mu, 2+\tau ; \omega_{R}} & +\left\|\partial_{y_{n}} u / y_{n}\right\|_{\mu, \tau ; \omega_{R}} \\
& \leq C\left[\left\|\mathcal{L}_{\alpha^{*}(0)} u\right\|_{\mu, \tau ; \omega_{R}}+\|u\|_{\mu, \tau ; \omega_{R}}\right] .
\end{aligned}
$$

Here $\underline{\omega}_{R}=\omega_{R} \cup\left(\partial \mathbf{R}_{+}^{n} \cap \partial \omega_{R}\right)($ See also (3-5)).

This proposition follows directly from Theorem 6-1 in $\S 6$ if $n \geq 3$. In case of $n=2$, we can show this proposition in the following way: In place of $\mathcal{L}_{\alpha^{*}(0)}$, we consider the operator $\mathcal{L}_{\alpha^{*}(0)}-\partial_{x_{0}}^{2}$, where $\partial_{x_{0}}$ is the derivative with respect to an auxiliary variable $x_{0}$. Then the Green function for this operator clearly exists and has the same properties, so that we can apply Theorem 5-1 again to prove Proposition 3-1 by using a suitable cut-off function in $x_{0}$ - space. 
Admitting this proposition for the moment, we shall prove Theorem 2-1 in the rest of this subsection ( See $\S 6$, for the proof of this proposition ). From this a priori estimate, we immediately have for any $v \in \Lambda_{2+\tau}^{2+\mu}\left(W_{R}\right) \cap \mathcal{E}^{\prime}\left(\underline{W}_{R}\right)$

$$
\|v\|_{2+\mu, 2+\tau ; W_{R}}+\left\|\partial_{y_{n}} v / y_{n}\right\|_{\mu, \tau ; W_{R}} \leq C\left[\left\|\mathcal{B}_{0} v\right\|_{\mu, \tau ; W_{R}}+\|v\|_{\mu, \tau ; W_{R}}\right] .
$$

Now we show that (3-17) implies Lemma 3-2 by virtue of a partition of unity, diffeomorphism and interporlation inequalities ( Theorem A-1), so that Theorem 2-1 follows. We take $\zeta(y) \in C_{0}^{\infty}\left(\mathbf{R}^{n}\right)$ such that $\operatorname{supp} \zeta \subset W_{2 r}, \zeta=1$ on $W_{r}$ and $\left.\partial_{y_{n}} \zeta(y)\right|_{y_{n}=0}=0$, where $r$ is an arbitrary number satisfying $0<r<R / 2$. For $u \in \Lambda_{2+\tau}^{2+\mu}\left(W_{R}\right) \cap \mathcal{E}^{\prime}\left(\underline{W_{R}}\right)$, we put $v=\zeta u$. Then we have

$$
\mathcal{B} v=\mathcal{B}_{0} v+\mathcal{B}_{1} v+\mathcal{B}_{2} v=\zeta(y) \mathcal{B} u+[\mathcal{B}, \zeta] u,
$$

and it follows from (3-17) that

$$
\begin{aligned}
& \|v\|_{2+\mu, 2+\tau ; W_{R}}+\left\|\partial_{y_{n}} v / y_{n}\right\|_{\mu, \tau ; W_{R}} \\
& \leq C\left[\|\zeta \mathcal{B} u\|_{\mu, \tau ; W_{R}}+\|[\mathcal{B}, \zeta] u\|_{\mu, \tau ; W_{R}}+\left\|\mathcal{B}_{1} v\right\|_{\mu, \tau ; W_{R}}\right. \\
& \left.\quad+\left\|\mathcal{B}_{2} v\right\|_{\mu, \tau ; W_{R}}+\|v\|_{\mu, \tau ; W_{R}}\right]
\end{aligned}
$$

Since $\partial_{y_{n}} \zeta(y)$ and the coefficients of the operator $\mathcal{B}_{1}$ are vanishing on the hyperplane $\left\{y_{n}=0\right\}$, there is a sufficiently small $\varepsilon_{0}>0$ such that if $0<r=R / 2<\varepsilon_{0}$ we have the desired estimate (3.10).

\section{§4. The proof of Theorem 2-2.}

In this subsection we shall prove Theorem 2-2, namely, the existence and the uniqueness of the solution to the homogeneous Neumann problem (N-P).

The Proof of Theorem 2-2. First we prove the uniqueness of the classical solution belonging to $\Lambda_{2+\tau}^{2+\mu}(\Omega)$. Auxiliarily we define

$$
W(\Omega)=\left\{u: u \text { is measurable and }\|u\|_{W}<\infty\right\},
$$

where

$$
\|u\|_{W}^{2} \equiv \int_{\Omega}\left(|\nabla u|^{2}+c u^{2}\right) \varphi^{\alpha} d x .
$$

Then $W(\Omega)$ becomes a Hilbert space and by $[W(\Omega)]^{\prime}$ we denote the dual space of $W(\Omega)$.

Lemma 4-1. Assume that $\tau>-(\alpha(x)+1) / 2$, and $\alpha(x)>-1$ on $\partial \Omega$. Then $f$ belongs to the dual space $[W(\Omega)]^{\prime}$ in a canonical way. if $f / \varphi^{\alpha} \in \Lambda_{\tau}^{\mu}(\Omega)$.

Proof of Lemma 4-1. Let $f / \varphi^{\alpha}$ be an element of $\Lambda_{\tau}^{\mu}(\Omega)$. Then it follows from Lemma A-2 that

$$
\left|f / \varphi^{\alpha}\right|_{\infty, \tau} \equiv \sup _{x \in \Omega}|f(x)| \varphi(x)^{(-\tau)_{+}-\alpha(x)}<+\infty .
$$


Hence we see $|f|^{2} \varphi^{-\alpha} \in L^{1}(\Omega)$ provided $2(-\tau)_{+}<\alpha(x)+1$ on $\partial \Omega$. This implies the assertion.

It follows from this lemma and Riesz' theorem that for every $f \in \Lambda_{\tau}^{\mu}(\Omega)$ there is a unique element $u \in W(\Omega)$ satisfying

$$
\int_{\Omega}[(\nabla u, \nabla v)+c u v] \varphi^{\alpha} d x=\int_{\Omega} f v d x, \quad \text { for any } v \in W(\Omega)
$$

Therefore the classical solution to (N-P) belonging to $\Lambda_{2+\tau}^{2+\mu}(\Omega)$ is also unique if there exists any.

Secondly we establish the existence of the solution. To do so we introduce a family of operators, namely,

$$
L_{\theta} u=-\varphi(x)^{-\theta \alpha(x)} \operatorname{div}\left(\varphi(x)^{\theta \alpha(x)} \nabla u\right)+c(x) u,
$$

with parameter $\theta \in[0,1]$. If $\alpha(x) \equiv 0$, then (N-P) is a variant of classical homogeneous Neumann problem. More precisely, it holds that

LEMMA 4-2. Assume that $\alpha(x) \equiv 0$ and $\max (-1+\mu,-1 / 2)<\tau<0$ on

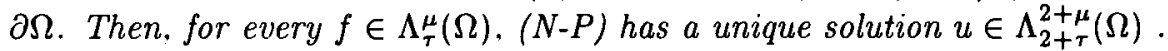

Proof of Lemma 4-2. In view of the classical theory, there is a Green function $G(x, y)$ to (N-P) with $\alpha(x)$ being identically 0 such that for every $f \in$ $C^{\mu}(\bar{\Omega}) .[G f](x) \equiv \int_{\Omega} g(x, y) f(y) d y \in C^{2+\mu}(\bar{\Omega})$ is a unique solution to (N-P). By Theorem 2-1 and Theorem 6-1, it is easy to see that the Green operator $G: C^{\mu} \rightarrow C^{2+\mu}$ can be extended to the bounded operator $G^{*}$ such that $G^{*}$ : $\Lambda_{\tau}^{\mu}(\Omega) \rightarrow \Lambda_{2+\tau}^{2+\mu}(\Omega)$ with the homogeneous Neumann condition. And clearly $u=G^{*} f, f \in \Lambda_{\tau}^{\mu}(\Omega)$ is a unique solution to (N-P), if $-1 / 2<\tau$. In particular if $\Omega=\mathbf{R}_{+}^{n}, G(x, y)$ coincides with $K_{0}(x, y)$, where $K_{0}(x, y)$ is the Green function to (N-P') (the homogeneous Neumann boundary value problem for the Laplacian in $\mathbf{R}_{+}^{n}$ in $\S 5$ ).

END OF THE PRoOF OF THEOREM 2-2. Let $F$ be the set of $\theta$ such that $L_{\theta}$ has the continuous inverse operator under the homogeneous Neumann condition. Then, $F$ is non-empty because $0 \in F$ by virtue of the above lemma. And Theorem 2-1 guarantees that $F$ is an open and closed subset of $[0,1]$. Therefore, $F=[0,1]$, in particular, $1 \in F$. Consequently, (N-P) has a unique solution for every $f$ satisfying the prescribed condition. Here we note that in each step of the reasoning, we need the restrictions on the values of $c(x), \alpha(x), \mu$ and $\tau$ (see $(2-3)$ and (2-5) ).

\section{§5. The Green kernel for the Neumann problem (N-P').}

In this section we shall define in $\mathbf{R}_{+}^{n}$ the Green function $K_{\alpha}(x, y)$ to (N-P') in $\S 2$ and describe its fundamental properties. 
Definition 5-1 : GREen FUnCtion For (N-P'). For $\alpha>0$, let us set $(5-1)$

$$
\begin{aligned}
& K_{\alpha}(x, y)=C_{\alpha}\left|x-y^{*}\right|^{-\alpha}|x-y|^{2-n} F\left(\frac{\alpha+2-n}{2}, \frac{\alpha}{2}, \alpha, \omega\right) \\
& \quad=C_{\alpha}\left|x-y^{*}\right|^{2-n-\alpha} F\left(\frac{n-2+\alpha}{2}, \frac{\alpha}{2}, \alpha, \omega\right) \\
& \quad=D_{\alpha} \int_{0}^{1}\left(|x-y|^{2}(1-\theta)+\left|x-y^{*}\right|^{2} \theta\right)^{-(n-2+\alpha) / 2}[\theta(1-\theta)]^{\alpha / 2-1} d \theta,
\end{aligned}
$$

where

$$
\begin{gathered}
y^{*}=\left(y^{\prime},-y_{n}\right), \quad \omega=1-\frac{|x-y|^{2}}{\left|x-y^{*}\right|^{2}}=\frac{4 x_{n} y_{n}}{\left|x-y^{*}\right|^{2}}, \\
C_{\alpha}=2^{\alpha-2} \pi^{-n / 2} \frac{\Gamma\left(\frac{n+\alpha-2}{2}\right) \Gamma\left(\frac{\alpha}{2}\right)}{\Gamma(\alpha)}, \quad D_{\alpha}=C_{\alpha} \frac{\Gamma(\alpha)}{\Gamma\left(\frac{\alpha}{2}\right)^{2}}
\end{gathered}
$$

$F(p, q, r, \omega)$ is a hypergeometric functions defined by

$$
F(p, q, r, \omega)=\frac{\Gamma(r)}{\Gamma(p) \Gamma(q)} \sum_{j=0}^{\infty} \frac{\Gamma(j+p) \Gamma(j+q)}{\Gamma(j+r)} \frac{\omega^{j}}{j !},
$$

and $F(p, q, r, \omega)$ satisfies the following equation:

$$
\omega(1-\omega) F^{\prime \prime}+(r-(1+p+q) \omega) F^{\prime}-p q F=0 .
$$

The kernel $K_{\alpha}(x, y)$ is not defined so far for $\alpha \leq 0$. However it can be continued to an entire function with respect to $\alpha \in(2-n, 0]$ if $n \geq 3$. To see this, choose and fix a nonnegative integer $m \in\left[0, \frac{n-2}{2}\right)$. And we assume that

$$
\alpha=2 \cdot p \leq 0, \quad \text { and } \quad-m-1<p \leq-m .
$$

Then by the analytic continuation with respect to $\alpha$, we immediately have

$$
K_{\alpha}(x, y)=I_{p}(x, y)+J_{p}(x, y)
$$

where

$$
\begin{aligned}
& I_{p}(x, y)=\frac{D^{*}(p)}{\Gamma(p)} \\
& \times \int_{0}^{1 / 2} \theta^{m+p} d \theta \int_{0}^{1} \frac{(1-\eta)^{m}}{m !}\left[f_{1}^{(m+1)}(\theta \eta)+f_{2}^{(m+1)}(\theta \eta)\right] d \eta \\
& J_{p}(x, y)=D^{*}(p) \frac{p(p+1) \cdots(p+m)}{\Gamma(p+m+1)} \sum_{l=0}^{m}\left[f_{1}^{(l)}(0)+f_{2}^{(l)}(0)\right] \frac{(1 / 2)^{p+l}}{l !(l+p)} \\
& D^{*}(p)=D(p) \Gamma(p)=2^{2 p} \pi^{-n / 2} \Gamma\left(\frac{n-2}{2}+p\right) \\
& f_{1}(\theta)=A(\theta)^{-q}(1-\theta)^{p-1}, f_{2}(\theta)=A(1-\theta)^{-q}(1-\theta)^{p-1} \\
& q=\frac{n-2}{2}+p, \quad A(\theta)=|x-y|^{2}+4 x_{n} y_{n} \theta
\end{aligned}
$$


For example,

$$
\begin{aligned}
& K_{0}(x, y)=C_{(0)}\left(|x-y|^{2-n}+\left|x-y^{*}\right|^{2-n}\right), \\
& K_{-2}(x, y)=C_{(-2)}\left(|x-y|^{4-n}+\left|x-y^{*}\right|^{4-n}\right. \\
& \left.-(n-4) x_{n} y_{n}|x-y|^{2-n}+(n-4) x_{n} y_{n}\left|x-y^{*}\right|^{2-n}\right), \text { if } n>4 .
\end{aligned}
$$

Here $C_{(0)}$ and $C_{(-2)}$ are positive numbers independent of $(x, y) \in \mathbf{R}_{+}^{n}$.

Then one can show the following fundamental properties of $K_{\alpha}(x, y)$. First of all we set

$$
u(x)=\left[K_{\alpha} f\right](x)=\int_{\mathbf{R}_{+}^{n}} K_{\alpha}(x, y) f(y) d y .
$$

Then $u$ is a solution of the problem (N-P'). More pricisely we have

Proposition 5-1. Assume that $\alpha>2-n$. Then $K_{\alpha}(x, y)$ is the Green function of the problem $\left(N-P^{\prime}\right)$. Namely, $u(x)=\left[K_{\alpha} f\right](x)$ satisfies the equation $L_{\alpha}=-\operatorname{div}\left(x_{n}^{\alpha} \nabla u\right)=f$ in $\mathbf{R}_{+}^{n}$ and $\left.\partial_{x_{n}} u\right|_{x_{n}=0}=0$, for any $f / x_{n}^{\alpha} \in C^{0}\left(\overline{\mathbf{R}_{+}^{n}}\right) \cap$ $\mathcal{E}^{\prime}\left(\underline{\mathbf{R}_{+}^{n}}\right)$.

Proof of Proposition 5-1. We shall establish this lemma assuming that $\alpha>0$. The proof in the excluded case follows in a similar way. By virtue of (5-3) we see that for any $(x, y) \in \mathbf{R}_{+}^{n} \times \mathbf{R}_{+}^{n}$ with $x \neq y$

$$
\begin{aligned}
L_{\alpha} K_{\alpha} & =-x_{n}^{\alpha}(\nabla \omega, \nabla \omega) \Phi F^{\prime \prime}(\omega)-2 x_{n}^{\alpha}(\nabla \omega, \nabla \Phi) F^{\prime}(\omega) \\
& +\Phi\left(L_{\alpha} \omega\right) F^{\prime}(\omega)+\left(L_{\alpha} \Phi\right) F(\omega) \\
= & \sigma\left[\omega(1-\omega) F^{\prime \prime}+\left(\alpha-\left(\alpha+\frac{n}{2}\right) \omega\right) F^{\prime}-\frac{\alpha(n-2+\alpha)}{4} F\right]=0
\end{aligned}
$$

where

$$
\begin{aligned}
& \sigma=\frac{x_{n}^{\alpha}(\nabla \omega, \nabla \omega)}{\omega(1-\omega)}, \Phi=C_{\alpha}\left|x-y^{*}\right|^{2-n-\alpha}, \\
& F=F\left(\frac{n-2+\alpha}{2}, \frac{\alpha}{2}, \alpha, \omega\right)
\end{aligned}
$$

By virtue of the homogeneity of the kernel $K_{\alpha}$, it is not difficult to see that $L_{\alpha} K_{\alpha}(x, y)=\delta(x-y)$ ( Dirac's delta function). Also from Theorem B-3, we see that $u$ satisfies the Neumann boundary condition.

After elementary calculations we see that the kernel $K_{\alpha}(x, y)$ obeys the following estimates. We only show a sketch of proofs in the case $\alpha>0$ (For the detailed proofs see [7]). Let us set

$$
g_{\alpha}(x, y)= \begin{cases}\left|x-y^{*}\right|^{-\alpha} & \text { if } \alpha \geq 0 \\ \left(x_{n}+y_{n}\right)^{-\alpha} & \text { if } 2-n<\alpha<0\end{cases}
$$


Proposition 5-2. Assume that $\alpha>2-n$. Then for any multi-index $\gamma$. there is a positive number $C(\gamma, \alpha)$ such that

$$
\begin{aligned}
& \left|\partial_{x}^{\gamma} K_{\alpha}(x, y)\right| / g_{\alpha}(x, y) \\
& \leq C(\gamma, \alpha)\left\{\begin{array}{l}
\log \left(2+\frac{\left|x-y^{*}\right|}{|x-y|}\right), \quad n=2 \cdot|\gamma|=0, \\
|x-y|^{2-n-|\gamma|}, \quad \text { otherwise. }
\end{array}\right.
\end{aligned}
$$

Here $C(\alpha, \gamma)$ is a positive number independent of each $(x, y) \in \mathbf{R}_{+}^{n} \times \mathbf{R}_{+}^{n}$.

Proposition 5-3. Assume that $\alpha>2-n$. Then, for any $\mu \in(0,1)$, there is a positive number $C(n, \alpha)$ such that a kernel $\partial_{n} K_{\alpha}(x, y)$ is a homogeneous function of degree $1-n-\alpha$ with respect to $(x, y)$ and satisfies the majoration:

$$
\left|\partial_{x_{n}} K_{\alpha}(x, y)\right| \leq C(n, \alpha) x_{n}^{\mu}|x-y|^{1-n-\mu} g_{\alpha}(x, y) .
$$

Here $C(n, \alpha)$ is independent of each point $(x . y) \in \mathbf{R}_{+}^{n} \times \mathbf{R}_{+}^{n}$.

Proof of Proposition 5-2 $(\alpha>0)$. We use the notations in (5-6). For any $x, y \in \mathbf{R}_{+}^{n}$ with $x \neq y$, we see that

$$
\left|\partial^{\gamma} K_{\alpha}(x, y)\right| \leq C(\alpha, \gamma) \int_{0}^{1} A(\theta)^{-|\gamma| / 2-q} \theta^{\alpha / 2-1} d \theta,
$$

where $\gamma$ is a arbitrary multi-index and $C(\alpha, \gamma)$ is a positive number independent of each $(x, y) \in \mathbf{R}_{+}^{n} \times \mathbf{R}_{+}^{n}$. Then, the desired estimate follows by a straightforward calculation.

Proof of Proposition 5-3 $(\alpha>0)$. Integration by parts gives us

$$
\left|\partial_{n} K_{\alpha}(x, y)\right| \leq C(n, \alpha) x_{n}|x-y|^{-n}\left|x-y^{*}\right|^{-\alpha} .
$$

Combining this with (5-11) we get the desired estimate.

\section{§6. An a priori inequality in $\mathbf{R}_{+}^{n}$ (Proof of Proposition 3-1 ).}

We shall estimate the solution to (N-P') in this subsection. First we define the function spaces on $\mathbf{R}_{+}^{n}$ analogous to $\Lambda_{\tau}^{\mu}(\Omega)$ and $\Lambda_{\tau}^{\mu}(\Omega)$ in $\S 2$.

Definition 6-1. Let $0<\mu<1, \tau \neq 0$, and let $f \in C\left(\mathbf{R}_{+}^{n}\right)$ be compactly supported in $\overline{\mathbf{R}_{+}^{n}}$. Then $f \in \Lambda_{\tau}^{\mu}\left(\mathbf{R}_{+}^{n}\right)$ if

$$
|f|_{\mu, \tau}=\sup _{x, y} \min \left(x_{n}^{\left.(\mu-\tau)_{+}, y_{n}^{(\mu-\tau)+}\right)} \frac{|f(x)-f(y)|}{|x-y|^{\mu}}<\infty .\right.
$$

Here $(\mu-\tau)_{+}=\max (0, \mu-\tau)$. For positive integer $k, f \in \Lambda_{\tau}^{k+\mu}\left(\mathbf{R}_{+}^{n}\right)$ if $D^{\gamma} f \in$ $\Lambda_{\beta-k}^{\mu}\left(\mathbf{R}_{+}^{n}\right)$ for any $\gamma$ with $|\gamma|=k$. We put $|u|_{k+\mu, \tau}=\sum_{|\gamma|=k}\left|D^{\gamma} u\right|_{\mu, \tau-k}$. We also set $\Lambda_{\tau}^{\mu}\left(\mathbf{R}_{+}^{n} ; l o c\right)=\left\{f \in C^{0}\left(\mathbf{R}_{+}^{n}\right) ; g f \in \Lambda_{\tau}^{\mu}\left(\mathbf{R}_{+}^{n}\right)\right.$, for any $\left.g \in C_{0}^{\infty}\left(\overline{\mathbf{R}_{+}^{n}}\right)\right\}$. 
$|\cdot|_{\mu, \tau}$ is then a norm on $\Lambda_{\beta}^{\mu}\left(\mathbf{R}_{+}^{n}\right)$, and $\Lambda_{\beta}^{\mu}\left(\mathbf{R}_{+}^{n}\right)$ is topologized as the inductive limit of Banach spaces exactly as $C^{\mu}\left(\mathbf{R}_{+}^{n}\right)$.

Auxiliarily we define

$$
C_{\tau}\left(\mathbf{R}_{+}^{n}\right)=\left\{f: f \in C\left(\mathbf{R}_{+}^{n}\right) \quad \text { and } \quad|f|_{\infty, \tau}<+\infty\right\},
$$

where $\quad|f|_{\infty, \tau} \equiv \sup |f(x)| x_{n}^{(-\tau)_{+}}$.

We also use the following notations. Let $S$ be a domain of $\overline{\mathbf{R}_{+}^{n}}$. Then we set

$$
\left\{\begin{array}{l}
|f|_{\infty, \tau ; S}=\sup _{x \in S}\left|x_{n}^{(-\tau)_{+}} f(x)\right|, \\
|f|_{\mu, \tau ; S}=\sup _{(x, y) \in S \times S} \min \left(x_{n}^{(\mu-\tau)_{+}}, y_{n}^{(\mu-\tau)_{+}}\right) \frac{|f(x)-f(y)|}{|x-y|^{\mu}} \\
\|f\|_{\mu, \tau ; S}=|f|_{\mu, \tau ; S}+|f|_{\infty, \tau ; S},\|f\|_{2+\mu, 2+\tau ; S}=\sum_{|\gamma| \leq 2}\left\|D^{\gamma} f\right\|_{\mu, \tau ; S} .
\end{array}\right.
$$

Let us recall that for $R>0$

$$
\begin{aligned}
& W_{R}=\left\{x=\left(x^{\prime}, x_{n}\right): 0<x_{n}<R,\left|x^{\prime}\right|<R\right\} \quad \text { and } \\
& \underline{W_{R}}=W_{R} \cup\left(\overline{W_{R}} \cap \partial \mathbf{R}_{+}^{n}\right) .
\end{aligned}
$$

Now we are in a position to state the main result in this section which was essentially used in $\S 3$ to prove Proposition 3-1 (a priori estimate of solutions ).

Theorem 6-1. Suppose that $\alpha>\max (-1,2-n), \max (-1,-1+\mu-\alpha)<$ $\tau \neq 0$, and $f / x_{n}^{\alpha} \in \Lambda_{\tau}^{\mu}\left(\mathbf{R}_{+}^{n}\right)$. Let $u=K_{\alpha} f$. Then,

(a) $\partial_{j} \partial_{k} u, \partial_{n} u / x_{n} \in \Lambda_{\tau}^{\mu}\left(\mathbf{R}_{+}^{n} ;\right.$ loc $)$ for $1 \leq j, k \leq n$, and $\partial_{n} \dot{u}=0$ on the boundary.

Moreover we have

(b) If $f / x_{n}^{\alpha} \in \Lambda_{\tau}^{\mu}\left(\mathbf{R}_{+}^{n}\right) \cap \mathcal{E}^{\prime}\left(W_{R / 2}\right)$, for some positive number $R$, then there is a positive number $C$ such that for $j, k=1,2, \ldots, n$, we have

$$
\left\{\begin{array}{c}
\left|\partial_{x_{j}} \partial_{x_{k}} u\right|_{\infty, \tau ; W_{R}}+R^{\min \left(\tau_{+}, \mu\right)}\left|\partial_{x_{j}} \partial_{x_{k}} u\right|_{\mu, \tau ; W_{R}} \\
\left.\leq C \| f /\left.x_{n}^{\alpha}\right|_{\infty, \tau}+R^{\min \left(\tau_{+}, \mu\right)}\left|f / x_{n}^{\alpha}\right|_{\mu, \tau}\right] \\
\left|\partial_{x_{n}} u / x_{n}\right|_{\infty, \tau ; W_{R}}+R^{\min \left(\tau_{+}, \mu\right)}\left|\partial_{x_{n}} u / x_{n}\right|_{\mu, \tau ; W_{R}} \\
\left.\leq C \| f /\left.x_{n}^{\alpha}\right|_{\infty, \tau}+R^{\min \left(\tau_{+}, \mu\right)}\left|f / x_{n}^{\alpha}\right|_{\mu, \tau}\right]
\end{array}\right.
$$

REMARK. (1) From Lemma A-2, it holds that for any $g \in \Lambda_{\beta-\alpha}^{\mu}\left(\mathbf{R}_{+}^{n}\right) \cap$ $\mathcal{E}^{\prime}\left(\underline{W_{R}}\right)$

$$
|g|_{\infty, \tau} \leq C|g|_{\mu, \tau} R^{\min \left(\tau_{+}, \mu\right)}
$$

where $C$ is a positive number independent of each $f$ and $R$.

(2) Since $\partial_{x_{n}} u / x_{n} \in \Lambda_{\tau}^{\mu}\left(\mathbf{R}_{+}^{n} ; l o c\right)$ by this Theorem, it follows from Lemma A-2 that $\left.\partial_{x_{n}} u\right|_{x_{n}=0}=0$, provided $\tau>-1$. 
(3) The condition $-1+\mu-\alpha<\tau$ is essentially needed to estimate the singular integrals.

Proof of Theorem 6-1. Now we shall establish Theorem 6-1 in a chain of auxiliary lemmas, which are corresponding to the estimates of the classical singular integral concerning the Newtonian potential. First we shall deal with the singular integrals involving the kernel $\partial_{x_{j}} \partial_{x_{k}}\left[y_{n}^{\alpha} K_{\alpha}(x, y)\right]$, for $j=1,2, \ldots, n$ and $k=1,2, \ldots, n-1$.

LEMMA 6-1. Assume that $\alpha>\max (-1,2-n)$. Let us set $u(x)=\left[K_{\alpha} f\right](x)$ $=\int_{\mathbf{R}_{+}^{n}} K_{\alpha}(x, y) f(y) d y$ for $F \equiv f / x_{n}^{\alpha} \in C_{0}^{\infty}\left(\overline{\mathbf{R}_{+}^{n}}\right) \cap \mathcal{E}^{\prime}\left(\underline{W_{R / 2}}\right)$. Then it holds that

$$
\begin{aligned}
\partial_{x_{j}} \partial_{x_{k}} u(x) & =\int_{W_{R}} \partial_{x_{j}} \partial_{x_{k}}\left(y_{n}^{\alpha} K_{\alpha}(x, y)\right)(F(y)-F(x)) d y \\
& -F(x) \int_{\partial W_{R}} \partial_{x_{j}}\left(y_{n}^{\alpha} K_{\alpha}(x, y)\right) \nu_{k}(y) d S_{y}
\end{aligned}
$$

Here $j=1,2, \ldots, n, \quad k=1,2, \ldots, n-1, \nu(x)=\left(\nu_{1}(x), \nu_{2}(x), \ldots, \nu_{n}(x)\right)$ is the outward normal to $\partial W_{R}$ and $d S_{x}$ indicates $(n-1)$-dimensional area element in $\partial W_{R}$.

Proof of Lemma 6-1. It suffices to use Green's formula. Note that the kernel $K_{\alpha}(x, y)$ is symmetric with respect to $x^{\prime}=\left(x_{1}, x_{2}, \ldots, x_{n-1}\right)$ and $y^{\prime}=$ $\left(y_{1}, y_{2}, \ldots, y_{n-1}\right)$, and $y_{n}^{\alpha} \partial_{j} K_{\alpha}(x, y), j=1,2, \ldots, n$ are locally integrable with respect to $y \in \overline{\mathbf{R}_{+}^{n}}$ provided $\alpha>\max (-1,2-n)$. For the precise proof, see Lemma 4.2 in [ $1 ;$ P. 55] for example.

In the representation formula (6-7), the second term is smooth in $\mathbf{R}_{+}^{n}$ and easily verified to satisfy the assertion. Hence it suffices to study the term of the form

$$
v(x)=\int_{\mathbf{R}_{+}^{n}} G_{\alpha}(x, y)(F(y)-F(x)) d y \quad \text { for } F(x)=f(x) / x_{n}^{\alpha},
$$

where

$$
\begin{aligned}
& G_{\alpha}(x, y)=y_{n}^{\alpha} \partial_{x_{j}} \partial_{x_{k}} K_{\alpha}(x, y) \\
& \quad \text { for } j=1,2, \ldots, n, k=1,2, \ldots, n-1 .
\end{aligned}
$$

To this end we choose non-negative smooth functions $\eta \in C_{0}^{\infty}\left(\underline{W_{R}}\right)$ and $\xi \epsilon$ $C_{0}^{\infty}\left(\mathbf{R}^{n}\right)$ so that $\eta \equiv 1$ on $W_{2 R / 3}$ and $\xi(x) \equiv 1$ for $|x| \leq 3 / 2, \overline{\xi(x)} \equiv 0$ for $|x| \geq 2$. Let us set $\xi_{\rho, x}(y)=\overline{\xi\left(\frac{y-x}{\rho}\right)}$ for $\rho>0, x \in W_{R / 2}$. Then we have the next lemma: 
LEMMA 6-2. Assume that $0<\mu<1, \max (-1,-1+\mu-\alpha)<\tau \neq 0, \alpha>$ $\max (-1,2-n)$ and $F(x) \in \Lambda_{\tau}^{\mu}\left(W_{R / 2}\right)$. Then, the function $v$ defined by $(6-2)$ belongs to $\Lambda_{\tau}^{\mu}\left(\mathbf{R}_{+}^{n} ;\right.$ loc $)$. More precisely, it holds that

$$
\begin{aligned}
& |v|_{\infty, \tau ; W_{R}}+R^{\min \left[\tau_{+}, \mu\right]}|v|_{\mu, \tau ; W_{R}} \\
& \quad \leq C\left[|F|_{\infty, \tau}+R^{\min \left[\tau_{+}, \mu\right]}|F|_{\mu, \tau}\right] .
\end{aligned}
$$

Here $C$ is positive number independent of each $F$.

Proof OF LemMa 6-2. We may assume that $F \in C_{0}^{\infty}\left(\overline{\mathbf{R}_{+}^{n}}\right) \cap \mathcal{E}^{\prime}\left(W_{R / 2}\right)$. First we estimate $|v|_{\infty . \tau: W_{R}}$. For $z=\left(x^{\prime}, R\right) \in \partial W_{R}$, we have

$$
\begin{aligned}
& |v(\cdot, R)|_{\infty, \tau ;\left|x^{\prime}\right|<R} \leq R^{(-\tau)+} \sup _{\left|x^{\prime}\right|<R}|v(z)| \\
& \leq C R^{(-\tau)+} \sup _{\left|x^{\prime}\right|<R}\left|\int_{W_{R / 2}} G_{\alpha}(z, y) F(y) d y\right| \\
& \leq C R^{(-\tau)+}|F|_{\infty, \tau} \int_{W_{R / 2} \cap\left\{y_{n}<R / 2\right\}} \frac{y_{n}^{(\tau)-} y_{n}^{\alpha} g_{\alpha}(x, y) d y}{|z-y|^{n}} \\
& \quad \text { (Proposition 5-1 and Lemma A-2) } \\
& \quad \text { (Homogeneity). }
\end{aligned}
$$

Then we have from Lemma A-2 (3)

$$
\begin{aligned}
& |v|_{\infty, \tau: W_{R}} \leq C\left[R^{(-\tau)_{+}}|v(z)|+|v|_{\mu, \tau ; W_{R}} R^{\min \left[\tau_{+}, \mu\right]}\right] \\
& \leq C^{\prime}\left[|F|_{\infty, \tau}+R^{\min \left[\tau_{+}, \mu\right]}|v|_{\mu, \tau ; W_{R}}\right] .
\end{aligned}
$$

Therefore all we have to do is to establish the Hölder estimate of $v$. To this end, we subdivide $v$ as follows.

$$
\begin{aligned}
& v(x)=v_{1}+v_{2}+v_{3}, \quad \text { where } \\
& v_{1}(x)=\int_{W_{R}} G_{\alpha}(x, y)(F(y)-F(x)) \xi_{x, \rho}(y) d y \\
& v_{2}(x)=\int_{W_{R}} G_{\alpha}(x, y)(F(y)-F(x))\left(\eta(y)-\xi_{x, \rho}(y)\right) d y \\
& v_{3}(x)=\int_{W_{R}} G_{\alpha}(x, y)(F(y)-F(x))(1-\eta(y)) d y .
\end{aligned}
$$

Clearly $v_{3}$ is smooth. So we shall establish Hölder estimates for $v_{1}$ and $v_{2}$. Take and fix a point $x \in W_{R / 2}$. It suffices from Lemma A-3 $(k=1 / 4)$ that we estimate them in such a ball

$$
B_{\rho}(x)=\{y:|y-x|<\rho\}, \quad 0<\rho \leq \frac{x_{n}}{4} .
$$


Choose a point $a \in \partial B_{\rho}(x)$ so that $|x-a|=\rho<x_{n} / 4$.

Estimate of $v_{1}$ AND $v_{2}$. From Theorem B-1 we immediately have

$$
\begin{aligned}
& \left|v_{1}(x)\right|=\left|\int_{W_{R}} G_{\alpha}(x, y)(F(y)-F(x)) \xi_{x, \rho}(y) d y\right| \\
& \leq C|F|_{\mu, \tau} \int_{W_{R}} \frac{y_{n}^{\alpha}\left(x_{n}^{(\tau-\mu)}-+y_{n}^{(\tau-\mu)}\right)}{|x-y|^{n-\mu}} g_{\alpha}(x, y) \xi_{x, \rho}(y) d y \\
& \leq C|F|_{\mu, \tau} \rho^{\mu} x_{n}^{(\tau-\mu)-} .
\end{aligned}
$$

And

$$
\left|v_{1}(a)\right| \leq C|F|_{\mu, \tau} \max \left(x_{n}^{(\tau-\mu)}-, a_{n}^{(\tau-\mu)_{-}}\right)|x-a|^{\mu}
$$

So that we have the desired estimate for $v_{1}$. With somewhat more calculations we see that $v_{2}$ satisfies the estimate (6-4). Hence we omit the detail.

END OF THE PRoOF of Theorem 6-1. For $F=f / x_{n}^{\alpha} \in \Lambda_{\tau}^{\mu}\left(\mathbf{R}_{+}^{n}\right)$, let us set $u(x)=\left[K_{\alpha} F\right](x)$. In the previous lemmas, we have already shown that $\partial_{x_{j}} u, \partial_{x_{j}} \partial_{x_{k}} u \in \Lambda_{\tau}^{\mu}\left(\mathbf{R}_{+}^{n} ; l o c\right), \quad j=1,2, \ldots, n, k=1,2, \ldots, n-1$. Therefore it suffices to show that $\partial_{x_{n}} u / x_{n}$ and $\partial_{x_{n}}^{2} u \in \Lambda_{\tau}^{\mu}\left(\mathbf{R}_{+}^{n} ; l o c\right)$. From the equation (N-P'), we have

$$
\partial_{x_{n}}\left(x_{n}^{\alpha} \partial_{x_{n}} u\right)=-f(x)-x_{n}^{\alpha} \Delta^{\prime} u(x), \quad \text { where } \quad \Delta^{\prime}=\sum_{k=1}^{n-1} \partial_{x_{k}}^{2}
$$

By virtue of Proposition 5-3, we have

$$
\begin{aligned}
& \frac{\partial_{x_{n}} u\left(x^{\prime}, x_{n}\right)}{x_{n}}=-\int_{0}^{1} h\left(x^{\prime}, x_{n} s\right) s^{\alpha} d s, \quad \text { where } \\
& h\left(x^{\prime}, x_{n}\right)=-\frac{f(x)}{x_{n}^{\alpha}}-\Delta^{\prime} u(x) \in \Lambda_{\tau}^{\mu}\left(\mathbf{R}_{+}^{n} ; l o c\right) .
\end{aligned}
$$

Since $h(x) \in \Lambda_{\tau}^{\mu}\left(\mathbf{R}_{+}^{n} ; l o c\right)$, the rest of the proof follows from the next lemma ( the proof is ommited).

Lemma 6-3. Assume that $\alpha>\max (-1,2-n), \max (-1,-1+\mu-\alpha)<$ $\tau \neq 0$, and $0<\mu<1$. Then $G(x)=\int_{0}^{1} g\left(x^{\prime}, x_{n} s\right) s^{\alpha} d s \in \Lambda_{\tau}^{\mu}\left(\mathbf{R}_{+}^{n} ; l o c\right)$ if $g \in \Lambda_{\tau}^{\mu}\left(\mathbf{R}_{+}^{n} ; l o c\right)$. More precisely it holds that, for $R>0$,

$$
|G|_{\mu, \tau ;, W_{R}} \leq C|g|_{\mu, \tau, W_{R}} \text {, and }|G|_{\infty, \tau ; W_{R}} \leq C|g|_{\infty, \tau: W_{R}}
$$

Here $C$ is a positive number independent of each $R$ and $g$. 


\section{$\S$ Appendix ( The modified Schauder space)}

Our main aim is to prove Proposition A which was stated in $\S 2$ and essentially used in the proof of Theorem 2-1. The proof is done in the following way: By virtue of a diffeomorphism and a partition of unity, the questions are essentially reduced to prove similar inequalities for $u$ with small support in a neghborhood of a boundary point, and this reduction can be done on the same lines as that in the classical theory. So it is easy to see that Proposition A directly follows from Lemmas below on the modified Schauder spaces on $\mathbf{R}_{+}^{n}$. More precisely, the assertions (1), (2) and (3) respectively follow from Lemma A-4, Lemma A-6, and Lemmas A-7 and A-8.

Proof of Proposition A. First of all we note that the similar spaces as $\Lambda_{\beta}^{\mu}\left(\mathbf{R}_{+}^{n}\right)$ were treated in C. R. Graham [3] and [4], and Lemmas A-1, A-2 and A-3 below are seen there. Therefore we omit the proofs of them.

\section{LEMMA A-1.}

$$
\begin{aligned}
& C^{\mu}\left(\mathbf{R}_{+}^{n}\right) \subset \Lambda_{\tau}^{\mu}\left(\mathbf{R}_{+}^{n}\right) \subset C^{\mu}\left(\mathbf{R}_{+}^{n} ; l o c\right) . \quad \text { And if } \tau \geq \mu, \text { then } \\
& \Lambda_{\tau}^{\mu}\left(\mathbf{R}_{+}^{n}\right)=C^{\mu}\left(\mathbf{R}_{+}^{n}\right) .
\end{aligned}
$$

Here $C^{\mu}\left(\mathbf{R}_{+}^{n} ;\right.$ loc $)$ is a local version of $C^{\mu}\left(\mathbf{R}_{+}^{n}\right)$ defined as

(A-2) $\quad C^{\mu}\left(\mathbf{R}_{+}^{n} ; l o c\right)=\left\{f \in C\left(\mathbf{R}_{+}^{n}\right) ; g f \in C^{\mu}\left(\mathbf{R}_{+}^{n}\right)\right.$ for any $\left.g \in C_{0}^{\infty}\left(\mathbf{R}_{+}^{n}\right)\right\}$.

LEMMA A-2.

(1) If $f \in \Lambda_{\tau}^{\mu}\left(\mathbf{R}_{+}^{n}\right)$ and $\tau<0$, then $f \in C_{\tau}$.

(2) If $\tau>0$ and $f \in \Lambda_{\tau}^{\mu}\left(\mathbf{R}_{+}^{n}\right)$, then $f\left(x^{\prime}, \cdot\right) \in C^{\min (\tau, \mu)}\left(\mathbf{R}_{+}^{1}\right)$, uniformly in $x^{\prime}=\left(x_{1}, \ldots x_{n-1}\right) \in \mathbf{R}^{n-1}$.

(3) If $f \in \Lambda_{\beta}^{\mu}\left(\mathbf{R}_{+}^{n}\right)$, then it holds that

$$
|f|_{\infty, \tau} \equiv \sup _{\mathbf{R}_{+}^{n}}|f(x)| x_{n}^{(-\tau)_{+}} \leq C|f|_{\mu, \tau} R^{\min \left(\tau_{+}, \mu\right)},
$$

where $R$ is a diameter of the support of $f$, and $C$ is a positive number independent of each $f$.

Lemma A-3. Assume that $0<\mu<1,0 \neq \tau \leq \mu$ and $0<k \leq 1$. Let $f \in C\left(\mathbf{R}_{+}^{n}\right)$ be compactly supported in $\overline{\mathbf{R}_{+}^{n}}$. If there is a positive number $C$ such that

$$
|f(x)-f(y)| \leq C\left(x_{n}^{\tau-\mu}+y_{n}^{\tau-\mu}\right)|x-y|^{\mu},
$$

for every $x, y$ with $\left|x_{n}-y_{n}\right| \leq k \min \left(x_{n}, y_{n}\right)$, then $f \in \Lambda_{\beta}^{\mu}\left(\mathbf{R}_{+}^{n}\right)$.

Lemma A-4 (Local version of Proposition A (1)). If $f \in \Lambda_{1+r}^{1+\mu}\left(\mathbf{R}_{+}^{n}\right), \tau>-1+\mu, \tau \neq 0$, then $f \in C^{\mu}$. Moreover we have

$$
|f|_{\mu, \mu} \leq C \rho^{\tau-+1-\mu}|f|_{1+\mu, 1+\tau}, \quad \rho=\sup _{x \in \operatorname{supp} f} x_{n},
$$


where $\tau_{-}=\min (\tau, 0)$ and $C$ is a positive number independent of each $f$ with support in a fixed compact set.

Proof of Lemma A-4. Take and fix a $z_{n} \in(\rho, 2 \rho)$. Then we see that

$$
\begin{aligned}
& \left|f\left(x^{\prime}, x_{n}\right)-f\left(x^{\prime \prime}, x_{n}\right)\right| \leq\left|\int_{z_{n}}^{x_{n}}\right| \partial_{t} f\left(x^{\prime}, t\right)-\partial_{t} f\left(x^{\prime \prime}, t\right)|d t| \\
& \quad \leq C\left|\partial_{x_{n}} f\right|_{\mu, \tau} \rho^{(\tau-\mu)_{-}+1}|x-y|^{\mu} .
\end{aligned}
$$

In a similar way we see

$$
\begin{aligned}
& \left|f\left(x^{\prime}, x_{n}\right)-f\left(x^{\prime}, y_{n}\right)\right| \leq\left|\int_{x_{n}}^{y_{n}}\right| \partial_{t} f\left(x^{\prime}, t\right)|d t| \\
& \leq C^{\prime}\left|\partial_{x_{n}} f\right|_{\mu, \tau}|x-y|^{\mu}\left(x_{n}^{1+\tau_{-}-\mu}+y_{n}^{1+\tau_{-}-\mu}\right) .
\end{aligned}
$$

So that we get, denoting by $C$ a positive number independent of each point $(x, y)$,

$$
|f(x)-f(y)| \leq C\left|\partial_{x_{n}} f\right|_{\mu, \tau}\left(\frac{\rho}{1+\rho}\right)^{1+\tau_{-}-\mu}(1+\rho)^{1+(\tau-\mu)-} .
$$

Therefore desired estimate follows.

LEMMA A-5. $\quad \log x_{n} \in \Lambda_{\tau}^{\mu}\left(\mathbf{R}_{+}^{n} ; l o c\right) \quad$ if and only if $\tau<0$.

Proof of Lemma A-5. In order to see $\log x_{n} \in \Lambda_{\beta}^{\mu}$, if and only if $\tau<0$, it suffices to show the inequality for some positive number $C$ :

$$
\left|\log \frac{x_{n}}{y_{n}}\right| \leq C\left(x_{n}^{\tau-\mu}+y_{n}^{\tau-\mu}\right)\left|x_{n}-y_{n}\right|^{\mu}, \quad \text { for } 0<x_{n}<y_{n} \leq 1 .
$$

If we put $x_{n}=t y_{n}$, this is equivalent to

$$
\frac{t^{\mu-\tau}}{1+t^{\mu-\tau}}|\log t||1-t|^{-\mu} \leq C \min \left[y_{n}^{\tau}, 1\right] \quad \text { for any } t, y_{n} \in(0,1]
$$

And this is clear if and only if $\tau<0$ for some positive number $C$ independent of each $y_{n}$.

From Lemma A-4 and A-5 we immediately have

Lemma A-6 ( Local version of Proposition A (2) ). If $f \in \Lambda_{\beta+1}^{\mu+1}\left(\underline{W_{R}}\right)$ with $-1+\mu<\tau<0$, then

(A-11) $\quad\left|f \cdot \log x_{n}\right|_{\mu, \tau} \leq C|f|_{1+\mu, 1+\tau}\left[R \log R+R^{1-\varepsilon}\right] \quad$ for any $0<\varepsilon<-\tau$.

Here $\underline{W_{R}}=\left\{x \in \overline{\mathbf{R}_{+}^{n}}:\left|x^{\prime}\right|<R, 0 \leq x_{n}<R\right\}$ and $C$ is a positive number independent of each $f$ and $R$.

Lastly the following interporlation inequalities are valid. 
Lemma A-7 ( Local version of Proposition A (3)).

Let $u \in \Lambda_{2+\tau}^{2+\mu}\left(\mathbf{R}_{+}^{n}\right) \cap \mathcal{E}^{\prime}\left(\underline{W_{R}}\right)$ with $\tau>-1+\mu, \beta \neq 0$. then we have for any $\varepsilon>0$.

$$
\begin{aligned}
& \left|\partial_{x_{j}} u\right|_{\infty, \gamma} \leq \varepsilon \cdot \sum_{j, k=1}^{n}\left|\partial_{x_{j} x_{k}}^{2} u\right|_{\infty, \gamma}+C(\varepsilon)|u|_{\infty, \gamma}, \text { for any } \gamma \leq \tau \\
& \left|\partial_{x,} u\right|_{\infty, \tau-\mu} \leq \varepsilon \cdot \sum_{j=1}^{n}\left|\partial_{x,} u\right|_{\mu, \tau}+C(\varepsilon)|u|_{\infty, \tau-\mu}, \\
& |u|_{\mu, \tau} \leq \varepsilon \cdot \sum_{j}^{n}\left|\partial_{x_{j}} u\right|_{\infty, \tau-\mu}+C(\varepsilon)|u|_{\infty, \tau-\mu}, \\
& |u|_{\infty, \tau-\mu} \leq R^{\max \left[0, \mu-(\tau)_{+}\right]}|u|_{\infty, \tau} .
\end{aligned}
$$

where $\mathcal{E}^{\prime}\left(W_{R}\right)$ is the set of distributions having compact support in $W_{R}=B_{R}(0) \cap$ $\overline{\mathbf{R}_{+}^{n}}$ and $\overline{C(\varepsilon)}$ is a positive number independent of each function $\bar{f}$.

Proof of Lemma A-7. Since these inequality can be established in a usual way. we omit the proof. For the detailed proof, see Horiuchi [7].

\section{References}

[1] D. Gilbarg, N.S. Trudinger, Elliptic partial differential equations of second order. 2nd ed., Berlin-Heidelberg-New York-Tokyo : Springer-Verlag 1983..

[2] C. Goulaouic, N. Shimakura, Regularité Hölderienne de certains problèmes aux limites elliptiques dégénérés, Anna. Scuola Norm. Sup. Pisa, serie IV 10 (1983), 79-108.

[3] C. R. Graham, The Dirichlet problem for the Bergman Laplacian I, Comm. Partial Differential Equations 8 No.5 (1983), 433-476.

[4] C. R. Graham. The Dirichlet problem for the Bergman Laplacian II, Comm. Partial Differential Equations 8 No. 6 (1983), 563-641.

[5] T. Horiuchi, Existence and uniqueness of classical solutions for certain degenerate elliptic equations of the second order, J. Math. Kyoto Univ. 24-3 (1984), 557-576.

[6] T. Horiuchi. On the Neumann problems for certain degenerate elliptic operators, Proc. of the Japan Acad. 69 Ser.A, No. 9 (1993), 372-376.

[7] T. Horiuchi. The Schauder approach to degenerate elliptic equation with homogeneous Neumann boundary condition I, Bul. Fac. Math. Sci. Ibaraki Univ. series A. 27 (1995), 7-32. 